\title{
PERFIL DOS MÉDICOS AUDITORES NO ESTADO DO RIO GRANDE DO SUL
}

\author{
Claus Michael Preger*, Israel Berger, Carlos Alberto Gonçalves de fonte, Helena Cesa Mascarello \\ Trabalho realizado no Curso de Pós-Graduação em Auditoria em Saúde, de Porto Alegre - \\ Universidade Gama Filho/RJ e Universidade Corporativa Unimed/MG.
}

RESUMO - Oвjetivo. 0 presente artigo analisa o perfil do médico auditor no Estado do Rio Grande do Sul, tomando por base o grupo de médicos que participou dos cursos de pós-graduação em Auditoria em Saúde, Fundação Unimed, nas cidades de Porto Alegre e Caxias do Sul.

Métodos. Apresentamos uma revisão histórica, estudo das leis e normas que regem a função de médico auditor e uma revisão bibliográfica sobre a auditoria médica no Brasil. Foi aplicado um instrumento fechado, no qual constavam dados pessoais e de formação profissional, assim como relativos à satisfação pessoal, saúde e bem-estar do entrevistado.

Resultados. Responderam ao questionário 71 médicos auditores com um total de 85 funções de auditoria médica em locais diversos de trabalho: $83,8 \%$ do sexo masculino, com faixa de idade preponderante de $4 \mathrm{I}$ a 50 anos (38\%), formados em média há 21,96 $+/-7,68$ anos, com tempo de atuação em auditoria médica de 7,24 +/= 6,62 anos, e 88,7\% possuem títulos universitários e/ou cursos de especialização médica nas mais diversas áreas de atuação. Do grupo pesquisado, $25,37 \%$ têm relação formal de emprego, $60 \%$ encontram-se satisfeitos como pessoa e como profissional, estando bem fisicamente. No grupo entrevistado, não existem médicos com menos de 33 anos de idade nem formados há menos de 7 anos.

Conclusöes. Fica evidente 0 alto grau de formação, especialização e de atualização dos médicos auditores entrevistados. 0 presente estudo contribuirá para que se possa estabelecer o perfil do médico auditor brasileiro.

Unitermos: Auditoria médica. Médico auditor. Perfil do médico auditor.

\section{INTRODUÇÃO}

A Auditoria Médica, apesar de sua importância - pois somente nos últimos anos tem sido valorizada -, é abordada em raros trabaIhos, e poucos livros sobre este tema foram, até hoje, publicados. Igualmente, os médicos que desempenham tal atividade, gradativamente foram dedicando mais tempo ao seu estudo e aperfeiçoamento.

Retrospectivamente, sabe-se que a avaliação da qualidade da assistência médica surgiu no início do século XX devido à crise na qualidade tanto do ensino médico como das instituiç̧ões de saúde nos Estados Unidos!.

A Association of American Medical College (AAMC) e a American Medical Association (AMA), em 1910, iniciaram controle no treinamento médico e no aparelhamento de hospitais. Em 1912, Codman, apud Carvalho², introduziu um sistema comparativo de resultados finais em cuja base os hospitais começaram a auditoria médica.

\footnotetext{
* Correspondência:

Rua Vitor Meireles, 149/40

Porto Alegre - RS - CEP: 90430-160

E-mail:cmpreger@uol.com.br

Tel: (51) 3330-4677 - Fax: (51) 3333-1307
}

Nos anos 50 , surgiram formas mais estruturadas que substituíram opiniões baseadas em avaliações subjetivas. A evolução natural dos diferentes tipos de controle culminou com a implantação da centralização do processamento dos dados hospitalares com programas do tipo Professional Activities Studies e Medical Audit Program ${ }^{3,4}$.

Até hoje, os programas visam a correta utilização dos leitos hospitalares e critérios mínimos de cuidados associando, definitivamente, a auditoria médica com a hospitalar.

No Brasil, o controle mais efetivo ocorreu na década de 60, quando a assistência médica passou por alterações mais profundas. As antigas Caixas de Previdência e Assistência Médica fundiram-se no Instituto Nacional de Previdência Social (INPS), seguindo-se a universalização da assistência médica.

Fato marcante ocorreu através do Decreto-lei 73/66, que criou o seguro-saúde para ser comercializado pelas Companhias de Seguro e também os planos de saúde, permitindo que profissionais se organizassem em instituições oferecendo serviços na forma de pré ou pós-pagamento.

O modelo assistencial estabelecia uma relação entre o usuário, o financiador e o prestador. Em razão de seus custos crescentes e inflacionários, abriu-se o espaço para a criação do sistema suplementar.

A avaliação dos sistemas de saúde se constituía em um elemento de maior importância na progressiva caracterização do que seja um sistema de saúde desejável e economicamente acessível ao país. Com o aprofundamento da municipalização de serviços no Brasil, financiadores, controladores do sistema e clientela tiveram que considerar a efetividade e a qualidade de centenas ou até milhares de sistemas praticamente independentes ${ }^{5}$.

$\mathrm{Na}$ década de 70, o Ministério da Previdência detectou supostas irregularidades na cobrança de contas médicas e hospitalares e iniciou um controle mais rigoroso nos serviços prestados através de auditagem médica e administrativa das contas. A Ordem de Serviço n..$^{\circ} 039.32$ da Secretaria de Assistência Médica do INPS, de 28 de dezembro de 1976, estabeleceu o controle formal e técnico dos serviços.

Pouco depois, na década de 80, o sistema suplementar igualmente estabeleceu sua auditoria médica, que gradualmente foi se consolidando como atividade necessária em todas as modalidades de assistência. 
O médico auditor passou a desempenhar importante papel de regulador entre a qualidade dos serviços prestados e seus respectivos custos, constituindo o fator que estabelece 0 equilíbrio.

Como vários interesses começaram a ser monitorados, os conflitos surgiram e, consegüentemente, várias infrações ao Código de Etica Médica (CEM).

Seguiram-se, então, vários pareceres tanto de Conselhos Regionais de Medicina (CRMs), como do Conselho Federal de Medicina (CFM) sobre como deveria proceder o Auditor e neles quase todos os artigos do CEM são citados, principalmente estabelecendo o que é permitido ou vedado aos médicos auditores e assistentes.

Alguns pareceres são específicos e emitidos em resposta às consultas dos Conselhos Regionais e do próprio Sistema Único de Saúde (SUS):

- Parecer no 02/94 do CFM - referente ao fornecimento do prontuário para auditoria

- Parecer no 03/94 do CFM - reporta-se à visita a pacientes hospitalizados, à beira do leito, por auditores ${ }^{7}$;

- Parecer no 21/94 do CFM - relaciona-se com o encaminhamento de prontuários médicos para a auditoria do SUS8;

- Parecer no 01/96 do CFM - refere-se ao impedimento ético de interferência na escolha terapêutica do médico assistente?;

- Parecer no 18/96 do CFM - dispõe sobre as atividades de fiscalização das ações e serviços de saúde e sobre a autonomia e limitações do médico auditor, responsável pela fiscalização das questões assistenciais ${ }^{10}$;

- Parecer no 20/96 do CFM - refere-se ao direito do paciente aos meios diagnósticos e à autonomia profissional do médico";

- Resoluçãa no | 466 de setembro de 1996 do CFM - relaciona as atividades do médico auditor ${ }^{12}$

- Parecer no 17/97 do CFM - reporta-se à interferência direta do auditor nas atividades médicas ${ }^{13}$;

- Parecer no II/99 do CFM - define autorização de exames pelo médico auditor, mudança ou solicitação de procedimentos, exame de pacientes e outras funções do auditor ${ }^{14}$.
Outrossim, a reorganização das atividades de controle e avaliação e de auditoria no âmbito do Ministério da Saúde foi estabelecida por Portaria assinada pelo Ministro José Serra em 19/08/9915.

A Resolução do Conselho Federal de Medicina | 466/96 foi revogada em 9 de março de 2001 , com a regulamentação da atividade de médico auditor por uma nova, a Resolução CFM $|6| 4 /\left.0\right|^{16}$, que, por sua vez, foi recentemente alterada em seu artigo $7^{017}$.

Em dezembro de 200 I, a Comissão Mista de Especialidades, constituída por representantes da Associação Médica Brasileira (AMB), do Conselho Federal de Medicina e da Comissão Nacional de Residência Médica, contemplou a Auditoria Médica e a Perícia Médica como Áreas de Atuação Especial - "modalidades de organização do trabalho médico, exercidas por profissionais capacitados para exercer ações médicas específicas, sendo derivadas e relacionadas com uma ou mais especialidades" -, portanto aptas a receber certificação específica pela $A M B^{18}$.

A atividade oficial e regulamentada de Auditoria Médica, por ser relativamente atual e estimulada pelas necessidades crescentes de controle das contas dos serviços hospitalares, médicos e complementares de diagnóstico e terapia do SUS e dos diversos planos de saúde privados, vem despertando a curiosidade científica de profissionais e estudantes das mais diversas áreas, resultando em trabalhos de pesquisa e de revisão ${ }^{19}$.

Portanto, para acompanhar as diversas providências e atuações no sentido de afirmar, aceitar e regulamentar a nova especialidade, é necessário traçar o perfil atual do médico auditor em todo território nacional: suas características de sexo, idade e tempo de exercício da medicina, seus cursos de aperfeiçoamento em diversas especialidades, suas características de saúde e as condições de valorização de seu trabalho por si próprio e por parte das instituições onde trabalha.

Pelas razões expostas, é o objetivo do presente trabalho estabelecer o perfil profissional do médico auditor, em primeira instância, no Estado do Rio Grande do Sul, tomando por base um grupo de médicos auditores participantes de cursos de pós-graduação em Auditoria em Saúde, da Universidade Gama Filho/RJ em parceria com a Universidade Corporativa UNIMED/MG, em Porto Alegre e
Caxias do Sul, e de outros médicos que também exercem essa especialidade.

\section{Métodos}

Utilizamos no presente trabalho a pesquisa de campo através da aplicação de um instrumento fechado que contemplou as principais características dos médicos auditores a serem estudadas.

O questionário foi dividido em três segmentos:

- Ficha funcional, com dados de identificação pessoal, como idade, sexo, tempo de atividade na medicina e em auditoria, local de atividade como auditor, o tipo de relação de trabalho, formaçãa e aperfeiçoamento médico em geral e em Auditoria Médica e informação sobre a possível aposentadoria;

- Dados de satisfação pessoal e valorização em que se procurou caracterizar o grau de satisfação intrínseca do médico, de satisfação no ambiente de trabalho, com a remuneração e o nível de valorização da atividade pela instituição empregadora através da promoção de cursos de aperfeiçoamento;

- Ficha médica, com dados sobre a existência de enfermidade prévia e atual, a permanência de valores alterados da bioquímica, o uso de medicação continuada, a necessidade de realização de alguma cirurgia, o número de faltas ao trabalho, sobre a sensação de bem-estar físico e, finalmente, sobre a possibilidade de encerramento de suas atividades profissionais.

Solicitamos aos médicos que não identificassem a si nem a instituição em que trabalhavam. O instrumento foi entregue e devolvido aleatoriamente, sem nenhuma ordem préestabelecida. Todos os questionários distribuídos foram devolvidos preenchidos total ou parcialmente.

O questionário foi aplicado aos médicos que já exerciam as tarefas de Auditoria Médica em alguma instituição de saúde, pública ou privada, inscritos nos cursos de pós-graduação em Auditoria em Saúde da Universidade Gama Filho/RJ, em parceria com a Universidade Corporativa UNIMED/MG, nas cidades de Porto Alegre e Caxias do Sul, RS. Também foi aplicado em médicos auditores sócios da Sociedade dos Médicos Auditores do Estado do Rio Grande do Sul (SOMAERGS), e em 
médicos auditores de outras instituições públicas e privadas, todos residentes em diversas cidades do Rio Grande do Sul.

Elaboramos a tabulação dos dados extraídos dos questionários e seu tratamento estatístico adotando as médias aritméticas e o desvio padrão. Anotamos também os valores mínimos, os máximos e a mediana.

Realizamos, ainda, a revisão histórica e a pesquisa bibliográfica sobre a Auditoria Médica em nosso país, embora restritas por tratar-se de uma atividade recente na área médica.

\section{Resultados e Discussão}

A análise dos dados obtidos com a aplicação do questionário aos médicos auditores permite apresentar e analisar os seguintes resultados:

\section{Dados Pessoais}

Número de funções de Auditoria Médica ou locais de trabalho (Tabela I)

Responderam ao questionário 7 I médicos auditores, dos quais 12 (16,90\%) tinham funções de auditores em dois ou mais locais de trabalho, sendo analisadas, portanto, 85 funções de auditoria médica;

Observa-se aqui também a tendência dos profissionais de medicina no Brasil de possuírem atualmente múltiplos empregos ou funções.

Idade média

A idade média é de 46,86 \pm 8,0 anos; a mediana, 48 anos; a menor, 33 anos; a maior, 68 anos.

\section{Tempo médio de atividade como médico}

O tempo médio de atividade como médico é de 21,96 \pm 7,68 anos; a mediana, 22 anos; o menor tempo, 7 anos; o maior tempo, 40 anos.

\section{Tempo médio na função de médico auditor}

O tempo médio na função de médico auditor é de 7,24 \pm 6,62 anos; a mediana, 5 anos; o menor tempo, I ano; o maior tempo, 27 anos.

Distribuição em faixas de idade (Tabela 2)

A faixa preponderante é a de $4 \mathrm{I}$ a 50 anos de idade (38,03\%). Os médicos acima de 60 anos constituem $8,45 \%$ do total. Não há médicos com menos de 30 anos de idade no grupo.

\begin{tabular}{|c|c|c|c|}
\hline \multicolumn{4}{|c|}{ Tabela I - Funções de auditoria médica } \\
\hline Funções & NoAuditores & $\mathrm{N}^{0}$ Funções & $\%$ \\
\hline $\begin{array}{l}\text { Única } \\
\text { Mútiplas }\end{array}$ & $\begin{array}{l}59 \\
12 *\end{array}$ & 59 & $\begin{array}{l}83,10 \\
16,90\end{array}$ \\
\hline Duas & (II) & 22 & .. \\
\hline Três & 0 & 0 & .. \\
\hline Quatro & (I) & 4 & \\
\hline Total de Auditores & 71 & & 100 \\
\hline Total de Funçōes & & 85 & \\
\hline
\end{tabular}

* Dos 12 médicos, II têm duas e um tem quatro funções em locais de trabalho distintos

\section{Tabela 2 - Distribuição da idade}

\begin{tabular}{lcc}
\hline Faixas & Número & $\%$ \\
\cline { 2 - 3 } Até 30 anos & 0 & 0 \\
$301-40$ & 17 & 23,94 \\
$40 \mid-50$ & 27 & 38,03 \\
$50 \mid-60$ & 21 & 29,58 \\
60 ou + & 6 & 8,45 \\
Total & 71 & 100 \\
\hline
\end{tabular}

Tabela 3 - Distribuição do tempo de atividade médica

\begin{tabular}{lcc}
\hline Faixas & Número & $\%$ \\
\cline { 2 - 3 } Até 5 anos & 0 & 0 \\
$51-10$ & 4 & 5,63 \\
$101-20$ & 26 & 36,62 \\
$201-30$ & 30 & 42,25 \\
30 ou + & 11 & 15,49 \\
Total & 71 & 100 \\
\hline
\end{tabular}

\begin{tabular}{lcc}
\hline \multicolumn{3}{c}{ Tabela 4 - Distribuição do tempo de auditoria } \\
\hline Faixas & Número & $\%$ \\
\cline { 2 - 3 } Até 5 anos & 30 & 42,86 \\
$51-10$ & 21 & 30,00 \\
$101-20$ & 13 & 18,57 \\
$201-30$ & 6 & 8,57 \\
30 ou + & 0 & 0 \\
Tota* & 70 & 100 \\
\hline
\end{tabular}

* um entrevistado não respondeu à questão

Distribuição em faixas de tempo de atividade médica (Tabela 3)

A faixa preponderante é a de 20 a 30 anos $(42,25 \%)$ de atividade na medicina. Não há médicos com menos de cinco anos de atividade, no grupo.

Os dados acima demonstram uma distribuição etária de $91,55 \%$ dos médicos auditores do grupo entre 30 e 60 anos, sendo os médicos da chamada Terceira Idade pouco representados e os jovens, inexistentes. São profissionais que acumularam sua experiência por vários anos antes de ingressarem na especialidade.

Distribuição em faixas do tempo de atividade em Auditoria (Tabela 4)

A faixa preponderante é a de até cinco anos (42,86\%). Não há médicos com mais de 30 anos de atividade em Auditoria no grupo.

A especialidade de Auditoria Médica, como foi apresentado na introdução do presente trabalho, é recente no Brasil, tanto é que 
Preger CM et al.

$72,86 \%$ dos médicos do grupo têm até 10 anos de atividade na mesma.

Distribuição porsexo

Dos 68 que responderam à questão, 57 $(83,82 \%)$ eram do sexo masculino e II $(16,18 \%)$, feminino. Não responderam à questão três entrevistados.

A preponderância do sexo masculino é de cerca de 5:1 sobre o feminino, não acompanhando portanto a tendência notória de ocupação do quadro dicente das faculdades de medicina, e conseqüentemente do contexto médico profissional, pelo segmento feminino.

\section{Aposentadorias}

Quatorze dos 7 I médicos eram aposentados (19,72\%), quatro $(5,63 \%)$ apenas como funcionários públicos, cinco $(7,04 \%)$ somente como autônomos e cinco $(7,04 \%)$ em ambas as situações.

\section{Relação de emprego}

Das 67 funções de auditoria médica analisadas, 17 (25,37\%) tinham relação formal com a empresa, com carteira de trabalho assinada, e 50 (74,63\%) tinham outro tipo de relação: sem carteira assinada, através de cooperativas médicas ou sócios de empresa de auditoria. Para as demais 18 funções, não houve resposta pelos entrevistados.

Ficam evidentes, também aqui, as mudanças no mercado atual de trabalho, onde cresce a relação sem vínculo empregatício. No último ano, somente 39\% da população ocupada, estudada na Pesquisa Mensal de Emprego pelo Instituto Brasileiro de Geografia e Estatística (IBGE), constitui-se de empregados com carteira assinada. Entre os médicos auditores do grupo estudado, este percentual é ainda menor: $25,73 \%{ }^{20}$.

Local de trabalho (Tabela 5)

Quanto ao local de trabalho na função de médico auditor, o maior número de funções são exercidas nas cooperativas ( $33=38,82 \%)$, nas autarquias $(16=18,82 \%)$ e nas empresas de autogestão $(12=\mid 4,12 \%)$.

Os dados mostram que as operadoras de planos de saúde representadas pelas cooperativas médicas, empresas de autogestão e de medicina de grupo constituem importantes fontes para o trabalho de auditoria médica.

\begin{tabular}{lcc}
\hline & Tabela 5 - Local de trabalho & \\
\hline Local & Número & $\%$ \\
\cline { 2 - 3 } Autarquia & 16 & 18,82 \\
Estatal & 3 & 3,53 \\
Cooperativa & 33 & 38,82 \\
Seguro-saúde & 3 & 3,53 \\
Medicina de grupo & 9 & 10,59 \\
Autogestão & 12 & 14,12 \\
Emp. de auditoria - sócio & 7 & 8,23 \\
Emp. de auditoria - empr. & 1 & 1,18 \\
Hospital & 1 & 1,18 \\
Funçöes & 85 & 100 \\
\hline
\end{tabular}

\section{Formação profissional}

Os 7I médicos entrevistados tinham a seguinte formação e especialização profissional:

- 48 (67,60\%) já haviam freqüentado um ou mais cursos de auditoria médica:

- 18 (25,35\%) já assistiram cursos correlatos (Administração Hospitalar, Gerenciamento em Saúde, Perícia Médica);

- 63 (88,73\%) possuem títulos de especialização médica e/ou universitários;

- 30 (42,25\%) pertencem a alguma associação de Auditoria ou Perícia Médica.

Chama a atenção o alto grau de formação, especialização e de atualização dos médicos auditores entrevistados. Ainda não foram conferidos títulos de especialidade em Auditoria Médica porque só recentemente o CFM e a AMB consideraram a Auditoria Médica uma Área de Atuação Médica Especial| ${ }^{18}$, ficando de regularizar a especialidade, e porque também só recentemente surgiram cursos de pós-graduação lato-sensu em Auditoria em Saúde.

\section{Índices de valorização e de satisfação no local de trabalho}

\section{Satisfação com seu trabalho na função de auditor}

Foram obtidos, para o número total de médicos que atuam em instituições privadas (66), os seguintes resultados quanto à satisfação com seu trabalho ou função:

- sentem-se muito satisfeitos ou satisfeitos: 44 (66,66\%);

- sentem-se satisfeitos em grau médio: 21 (31,82\%);

- sentem-se pouco satisfeitos ou insatisfeitos: I (I,5l\%).
Nas instituições estatais ou autarquias (19):

- sentem-se muito satisfeitos ou satisfeitos: 7 (36,84\%);

- sentem-se satisfeitos em grau médio: 7 (36,84\%);

- sentem-se pouco satisfeitos ou insatisfeitos: $5(26,31 \%)$.

\section{Satisfação com a remuneração na função}

Foi considerado o número total de funções ou locais de trabalho (85):

Nas instituições privadas (66):

- sentem-se satisfeitos: 24 (36,36\%);

- sentem-se satisfeitos em grau médio: 34 (5I,52\%);

- sentem-se pouco satisfeitos ou insatisfeitos: $8(12,13 \%)$.

Nas instituições estatais ou autarquias (19):

- sentem-se satisfeitos: 2 (10,53\%);

- sentem-se satisfeitos em grau médio: 9 (47,37\%);

- sentem-se pouco satisfeitos ou insatisfeitos: $8(42,10 \%)$.

Não há médicos no grupo que se sintam muito satisfeitos com sua remuneração.

Por outro lado, se evidencia que a satisfação com seu trabalho profissional e/ou com sua remuneração é maior entre os médicos vinculados a instituições privadas do que entre os servidores públicos, o que é perfeitamente compreensível levando em conta a desproporção ocorrida nos últimos anos entre os índices de inflação nacional e o reajuste nos vencimentos do funcionalismo público.

\section{Aperfeiçoamento profissional promovido pela instituição a que pertencem}

Foram consideradas as respostas dos médicos no número total de funções (85): 
- tiveram cursos atuais patrocinados pelas empresas: 34 (40\%);

- tiveram patrocinados cursos anteriores: 38 (44,71\%);

- seus colegas de trabalho também tiveram cursos patrocinados: 35 (41, 18\%);

- nenhum curso promovido: 35 (41, 18\%).

As cooperativas médicas destacaram-se com o patrocínio dos cursos atuais de Auditoria Médica, de cursos anteriores e de cursos em geral a outros colegas de trabalho, demonstrando preocupação, portanto, com o aprimoramento continuado dos médicos a elas vinculados:

- patrocínio dos cursos atuais: 29 (87,88\%);

- patrocínio de cursos anteriores: 25 (75,76\%);

- patrocínio de outros colegas de trabalho: 27 (81,82\%);

- nenhum curso promovido: I (3,03\%).

\section{Ficha médica}

$\bigcirc$ perfil de saúde do médico auditor do grupo aqui apresentado denota a preocupação do médico com a sua saúde, e os percentuais de enfermidades, bioquímica alterada e utilização de medicação de uso continuado apontam para ao acerto desta preocupação. Raros são os artigos nacionais e do exterior publicados com referência às enfermidades que atingem o médico ${ }^{21,22}$, indicando uma linha de pesquisa a ser desenvolvida futuramente.

\section{Conclusóes}

- médico auditor no Estado do Rio Grande do Sul é um profissional predominantemente do sexo masculino, na faixa etária entre 40 e 50 anos, formado há 22, I \pm 7,72 anos, com títulos de especialização e/ou universitários, tendo já freqüentado um ou mais de um curso nesta área.

Seu vínculo de trabalho é preferentemente informal, atuando como auditor há 7,24 \pm 6,62 anos.

Os médicos entrevistados mostraram estar satisfeitos com sua atividade, mais na instituição privada do que na pública, e sua remuneração equipara-se a dos outros médicos não auditores.

Com referência ao estado de saúde, a maioria sente-se bem fisicamente, apesar de a quarta parte dos pesquisados relatar alguma moléstia importante e utiliza medicamentos de uso prolongado.

\begin{tabular}{lccc}
\hline \multicolumn{3}{c}{ Tabela 6 - Sinopse da ficha médica } \\
\hline Aplicação do questionário & $\frac{\text { Número }}{7}$ & & Percentual \\
Resposta ao questionário & 67 & 100 \\
Relatam enfermidade prévia importante* & 22 & 32,83 \\
Enfermidades atuais* & 17 & 25,37 \\
Apresentam bioquímica alterada & 08 & 11,94 \\
Utilizam medicamento de uso continuado & 20 & 29,85 \\
Probabilidade de cirurgia em curto espaço de tempo & 01 & 1,49 \\
Sentem-se muito bem ou bem fisicamente & 62 & 92,53 \\
Intenção de suspender atividades profissionais nos próx. cinco anos & 03 & 4,47 \\
\hline
\end{tabular}

* Cardiopatia, Hipertensão, Espondilopatia, Nefropatia, Pneumopatia, etc.

No grupo entrevistado não existem médicos com menos de 33 anos de idade nem formados há menos de 7 anos, o que demonstra a tendência dos médicos auditores serem profissionais que acumularam sua experiência por vários anos.

A maioria tem menos de 30 anos de formada (84,5\%), menos de 60 anos de idade ( $91,5 \%$ ) e, embora cerca de $20 \%$ dos médicos estejam aposentados, permanecem em plena atividade profissional.

As cooperativas médicas investem no aprimoramento profissional de seus médicos auditores mais do que as outras operadoras de planos de saúde.

Fica evidente o alto grau de formação, especialização e de atualização dos médicos auditores entrevistados.

O presente estudo contribuirá para que se possa estabelecer o perfil do médico auditor brasileiro, uma vez que se trata de atividade médica só recentemente regulamentada no Brasil.

\section{Conflito de interesse: não há.}

\section{SUMMARY}

Profile of a medical auditing group IN the state of Rio Grande do Sul

Background. This paperaims to determine the profile of the medical auditor in the State of Rio Grande do Sul, Brazil, based upon a group of physicians who participated in the postgraduation courses of Unimed Foundation Health Auditing, in the cities of Porto Alegre and Caxias do Sul, together with other professionals performing the same activity in the State of Rio Grande do Sul.

Methods. A historical review, a study of the laws and norms that guide the role of the
Medical Auditor is presented as well as a review of the literature on medical auditing in Brazil. A non identifiable questionnaire was administered, where questions on personal data and professional training were made, as well as others related to personal satisfaction, health and well- being.

Results. The questionnaire was answered by 71 Medical Auditors, with a total of 85 positions in medical auditing at several workplaces. $83.8 \%$ are male, ages ranging from 4 I to 50 years (38\%), on the average they had been graduated for $21.96+/-7.68$ years, performing medical auditing activities for 7.24 +/- 6.62 years and $88.7 \%$ hold a medical academic title and/or medical specialization courses in several areas. In the group researched, $25.37 \%$ are formally employed $60 \%$ are personally and professionally satisfied and are physically well. In the interviewed group, there are no physicians under 33 years of age nor any graduated for less than 7 years.

Conclusions. A high level of medical education, specialization and updating is evidentamong the Medical Auditors that were interviewed. Data from the present study will contribute for the definition of the Brazilian Medical Auditor's Profile. [Rev Assoc Med Bras 2005; 5I(2): 87-92]

Key words: Medical auditing. Medical auditor. Medical auditor profile.

\section{REFERÊNCIAS}

I. Malafaia O, De Paola D, Wittig EO. Auditoria médica e suas implicações legais. Rev Assoc Med Bras 1983;29:26-7.

2. Carvalho MP. Auditoria médica em hospitais escola. Residência Médica 1981;13:1 .

3. Butler J, Quinlan JW. Internal audit in the department of medicine of a community hospital. Two years experience. JAMA 1958; 167:567-72. 
4. Lembcke, PA. Evolution of the medical audit. JAMA 1967; 199: I | I-8.

5. Azevedo, AC. Avaliação de desempenho de serviços de saúde. Rev Saúde Pública 1991; 25:64-71.

6. Conselho Federal de Medicina. Brasília. Processo-Consulta 02/94. Relator Nei Moreira Silva. 13 jan 1994. Disponível em: http://www.cfm.org.br.

7. Conselho Federal de Medicina. Brasília. Processo-Consulta 03/94. Relator Nei Moreira Silva. 13 jan 1994. Disponível em: http://www.cfm.org.br.

8. Conselho Federal de Medicina. Brasília. Processo-Consulta 21/94. Relator Nei Moreira Silva. 12 ago 1994. Disponível em: http://www.cfm.org.br.

9. Conselho Federal de Medicina. Brasília. Processo-Consulta 0I/96. Relator Edson Oliveira Andrade. 12 abr 1996. Disponível em: http://www.cfm.org.br.

10. Conselho Federal de Medicina. Brasília. Processo-Consulta 18/96. Relator Paulo Eduardo Behrens. I0 set 1996. Disponível em: http://www.cfm.org.br.
11. Conselho Federal de Medicina. Brasília. Processo-Consulta 20/96. Relator José Ricardo Holanda Cavalcanti. 10 abr 1996. Disponível em: http://www.cfm.org.br.

12. Conselho Federal de Medicina. Brasília. Resolução I 466/96. RelatorWaldir Paiva Mesquita. 17 set 1996. Disponível em: http:// www.cfm.org.br.

13. Conselho Federal de Medicina. Brasília. Processo-Consulta 17/97. Relator Paulo Eduardo Behrens. 7 maio 1997. Disponível em: http:// www.cfm.org.br.

14. Conselho Federal de Medicina. Brasília. ProcessoConsulta I I/99. Relator Paulo Eduardo Behrens. 7 abr 1999. Disponível em: http://www.cfm.org.br.

15. Ministério da Saúde. Portaria 1069/99. Diário Oficial da União, Brasília (DF) 199920 ago.

16. Conselho Federal de Medicina. Brasilia. Resolução | 6 | 4/200 I. Diário Oficial da União, Brasília (DF) 20013 mar; Seção I:6. Disponível em: http://www.cfm.org.br.

17. Conselho Federal de Medicina. Brasília. Resolução I6 |4/200 I. Retificação. Diário Oficial da União (DF) 200 I I 0 abr. Disponível em: http://www.cfm.org.br.
18. Conselho Federal de Medicina. Brasília. Resolução 1634/2002. Comissão Mista de Especialidades. II abr 2002. Disponível em: http://www.cfm.org.br

19. Milani CZ. Auditoria médica [monografia]. Joaçaba: Universidade do Oeste de Santa Catarina, Campus de Joaçaba; 1998.

20. IBGE - Pesquisa mensal de emprego. Brasília. Disponível em: http://www.IBGE.gov.br.

21. Dioguardi GS, Pimenta J, Knopliich J, Ghorayeb N, Ramos LR, Giannini SD. Fatores de risco para doenças cardiovasculares em médicos: dados preliminares do projeto VIDAM da Associação Paulista de Medicina. Arq Bras Cardiol 1 994;62:383-8.

22. Pattani A, Constantinovici N, Williams S. Who retires early from the NHS because of ill health and what does it cost? A national cross sectional study. BMJ 200 I;322:208-9.

Artigo recebido: 03/07/03 Aceito para publicação: 13/10/04

\section{www.ramb.org.br}

A Ramb disponibiliza agora um novo serviço para os nossos colaboradores:

0 envio de artigos pela internet. Através do nosso publicador, que agiliza o fluxo de submissão com mais rapidez e versatilidade, você poderá enviar seu artigo para publicação na Ramb. Experimente: é muito mais cômodo, muito mais rápido e você ainda poderá acompanhar on line o curso de sua apreciação. Acesse www.ramb.org.br 